\title{
El papel de las redes sociales en la participación organizada de los jóvenes en Nicaragua
}

The role of social media in the organized participation of young people in Nicaragua

\author{
Luis Jara \\ Universidad Autónoma de Nuevo León \\ Orcid https://orcid.org/oooo-0oo1-9193-2804 \\ ljarapc@uanl.edu.mx
}

Resumen: El uso de redes sociales figura como la causa asumida que impacta en la organización y participación ciudadana de los jóvenes en Nicaragua. Éstos constituyen, en gran medida, la estructura poblacional del país centroamericano y la participación del conjunto resulta fundamental para sostener la lógica de la democracia. El estudio científico es de corte cualitativo y se utiliza la entrevista a profundidad como herramienta de recolección datos. Así, se busca conocer el papel de las redes sociales digitales en la actividad de los jóvenes nicaragüenses organizados en movimientos universitarios e identificar la relación entre el uso de redes sociales, el interés político y la participación del ciudadano joven en los asuntos públicos en Nicaragua. Los resultados de la investigación destacan el alcance e inmediatez de las redes y como el uso de las redes aumenta el interés en lo político, siempre y cuando, este tema se encuentre en el área de interés del individuo que interactúa en la plataforma digital.

Palabras clave: Interés político, joven, participación ciudadana, redes sociales, movimientos universitarios

\begin{abstract}
The use of social networks appears as the assumed cause that impacts the organization and citizen participation of young people in Nicaragua. These constitute, to a large extent, the population structure of the Central American country and the participation of the whole is fundamental to sustain the logic of democracy. The scientific study is qualitative and the indepth interview is used as a data collection tool. Thus, it seeks to know the role of digital social networks in the activity of Nicaraguan young people organized in university movements and to identify the relationship between the use of social networks, political interest and the participation of young citizens in public affairs in Nicaragua. The results of the research highlight the scope and immediacy of the networks and how the use of the networks increases interest in politics, as long as this topic is in the area of interest of the individual who interacts on the digital platform.
\end{abstract}

Keywords: Political interest, youth, citizen participation, social networks, university movements

Fecha de recepción: 22/11/2021

Fecha de aprobación: 21/12/2021

Cómo citar este artículo: Jara, L. (2021). El papel de las redes sociales en la participación organizada de los jóvenes en Nicaragua. Revista de Comunicación Política, 3, e210303. https://doi.org/10.29105/rcp3-3 


\section{Introducción}

Para introducir el estudio relacionado con participación ciudadana, juventud y redes sociales digitales tales como Facebook (la de mayores usuarios), resulta necesario subrayar que la imposición de la voluntad gubernamental, la censura y la radicalización de las ideologías políticas limita los espacios físicos (offline) de participación ciudadana (Jara \& Saldierna, 2020). Tal situación exige que se ajuste la intención participativa a un panorama globalizador que evidencia la estrecha relación entre interacción social y redes digitales. Así, por ejemplo, la Primavera Árabe de 2010 es resultado de la ausencia de libertad y constante dominación o violación de los derechos humanos; hecho denunciado y demostrado por la cibercultura (Rihawi Pérez, 2017).

En ese sentido, Valencia y García (2014) aseguran que "nadie concibe la vida sin las prácticas en red, por medio de las cuales se mira el mundo y desde las cuales muchos se hacen visibles" (p. 83). Mas aún, la ciudadanía, en su día a día, se encuentra expuesta a la información presente en las redes sociales, y tal exposición, activa o incidental, fomenta la participación ciudadana (Gil de Zúñiga et al., 2015) y fortalece la lógica de la democracia. Por tanto, la introducción de la variable digital promueve un aumento en la capacidad o alcance de la participación ciudadana. Así mismo, el uso de redes sociales se concibe como una variante moderadora de la relación político-social.

Cabe destacar que, en Nicaragua, aún son escasas las investigaciones respecto al papel de las redes sociales digitales en la organización, participación e interés político en los jóvenes que constituyen, aproximadamente, más del 50\% de la población en el país centroamericano (Banco Mundial [BM], 2020; Muñoz \& Narváez, 2018). En consonancia con lo anterior, la presente investigación resulta ser un tema de estudio importante por su impacto social y su aportación al debate teórico (Bacallao-Pino, 2010). Ejemplos como la revolución pingüina en Chile en 2006, el movimiento chileno en 2011 y el \#YoSoy132 en México en 2012, ponen de manifiesto la importancia de la juventud organizada y el uso de las redes sociales en defensa de los derechos y la participación ciudadana.

En Nicaragua, de acuerdo con Rueda Estrada (2018), las manifestaciones en espacios públicos se encuentran alejadas del control ciudadano. Por tanto, se contradice el derecho y la práctica debido a que la capacidad para discutir sobre la dirección del gobierno es muy restringida (Martí I Puig, 2016). Sin embargo, Castro (2018) considera que "la crisis política y social que estalló en abril en Nicaragua [...] resaltó la fuerte incidencia de las comunicaciones, en general, del Internet y las redes sociales, en particular, en la configuración del espacio político y la opinión pública" (p. 169).

Así, el presente estudio científico exhibe como propósito, conocer el papel de las redes sociales digitales en la participación organizada del ciudadano joven nicaragüense y el cambio que puede 
ocasionar el servicio de las redes sociales en el interés político, en aras de abonar al debate teórico y fortalecer la comprensión del fenómeno en el país.

\section{Participación ciudadana, juventud y espacio digital}

De manera puntual, Serrano (2015), asegura que "la participación ciudadana es aquella donde la sociedad posee una injerencia directa con el Estado; asimismo, tiene una visión más amplia de lo público. Esta participación está muy relacionada con el involucramiento de los ciudadanos en la administración pública” (p. 96). Conviene subrayar que la participación de los miembros activos de un Estado, permite identificar de manera concreta los problemas que afectan a la sociedad, encontrar respuestas en conjunto y orientar las voluntades que se encuentran dispersas en el sistema político; todo, a través de un único esfuerzo (Merino, 2016).

En ese sentido, se estima como sustancial garantizar la convivencia pacífica y el respeto a los derechos humanos, en especial los derechos políticos de las minorías, tomando en cuenta que el idealismo se ausenta de la práctica interna de las entidades estatales y se incumple la regla donde "los más" tienen el derecho a mandar, pero respetando los derechos de "los pocos" (Sartori, 2009).

Ahora bien, ante la realidad de la relación entre los tomadores de decisiones (decision makers) y la ciudadanía, sumado a la reducción de los espacios de participación, las redes sociales digitales fungen como un nuevo espacio por el cual el ciudadano joven y organizado, por ejemplo, a través de movimientos universitarios, puede protestar y demandar un cambio. Si bien se comenta sobre la pasividad, la falta de consumo de información política o ausencia de compromiso por parte de la juventud (Echeverria, 2011), también resulta necesario considerar que a pesar de que los jóvenes no demuestran a viva voz un interés en la participación política convencional, éstos están conectados con la esfera pública, y lo están en parte gracias al uso de Facebook, YouTube, Twitter, por mencionar algunas plataformas digitales (Valenzuela et al., 2012).

Además, relacionado con la importancia de las redes sociales digitales, Vaccari et al (2015) aclaran que en un inicio se pensó que las plataformas digitales únicamente reforzaban los patrones de participación y desigualdad existentes en la sociedad. Sin embargo, se ha demostrado que la dinámica digital construye un espacio de debate e interacción entre gobernantes y gobernados, lo que permite la adopción de medidas que contribuyen a resolver -hasta cierto punto- los conflictos o necesidades sociales (Cabalin et al., 2014; Sola-Morales \& Bacallao-Pino, 2016).

Por su parte, Dorantes y Aguilar (2016) destaca que el uso de lo digital, sumado al carácter multidireccional de las nuevas tecnologías, genera un equilibrio útil que se traduce en un alto nivel 
de impacto y horizontalidad en las relaciones en el sistema político. Precisamente, las manifestaciones centradas, en primera instancia, en la transmisión de información a través de nuevas tecnologías, permiten a la ciudadanía tomar decisiones acertadas, lo que contribuye a construir un proceso de participación con bajos índices de violencia, y al mismo tiempo, afectar la inclinación de la opinión pública (Castells et al., 2007). En el caso particular de los jóvenes, Ito (2008, citado en García \& del Hoyo, 2013) comenta que:

la participación online ha proporcionado a los jóvenes, oportunidades para discutir temas sociales o políticos, aprender sobre diferentes aspectos predominante en la sociedad, valores y experiencias vitales, aumentar su capital social, así como para desarrollar conocimientos sobre las normas de interacción entre grupos que pueden facilitarle su participación política y cívica en su vida fuera de las redes sociales. (p. 115)

Se debe agregar que, Musso (2004) asegura que las redes sociales son "una estructura de interconexión inestable, compuesta por elementos de interacción, y cuya variabilidad obedece a alguna regla de funcionamiento" (p. 31). Existe, empero, en esa inestabilidad, la libertad suficiente que consiente la autorrealización de los jóvenes. En efecto, la era digital permite presentar con mayor diligencia las demandas de los jóvenes y dar respuesta a sus intereses.

En el caso de Nicaragua, se estima que a los jóvenes no les interesa participar e incidir en la toma de decisiones (Osorio Mercado \& Serra Vásquez, 2012; Telléz, 2009). Sin embargo, desde el año 2018, los jóvenes organizados, con la intención de promover una transformación social, actúan como catalizadores que agrupan fuerzas, opiniones y emociones en lo digital. De ahí que, ante los cambios generacionales, exista la obligación de tomar acciones importantes utilizando nuevas formas de comunicación social que permitan la participación activa del ciudadano en los asuntos públicos.

\section{Objetivos y preguntas de investigación}

La presente investigación científica expone como objetivo principal conocer el papel de las redes sociales digitales en la organización y participación de los jóvenes. Por lo cual, se persigue identificar la relación entre el uso de redes sociales, el interés político y la participación del ciudadano joven en los asuntos públicos en Nicaragua. 
Así, con base en la revisión de literatura previa, se formulan las siguientes preguntas de investigación que se responderán con los datos del estudio:

PI1. ¿Cuál es el rol que desempeña el uso de redes como generador de participación ciudadana en los jóvenes nicaragüenses?

PI2. ¿Cuál es el papel de las redes sociales en el interés político en los jóvenes nicaragüenses?

\section{Metodología}

El presente estudio utiliza una metodología de corte cualitativo. Como tal, se aplican entrevistas en profundidad, de acuerdo con el criterio de estructuración, e, individuales, según el número de participantes (Folgueiras Bertomeu, 2016). La entrevista, es un instrumento que permite sistematizar el discurso de los informantes calificados (Jara \& Saldierna, 2020; Troncoso Pantoja \& Amaya Plascencia, 2017; Pérez Rodríguez et al., 2021) y relacionar la voz con las características particulares y experiencias de los participantes (Fontana \& Frey, 2005, citados por Vargas-Jiménez, 2012; Jara \& Saldierna, 2020).

Tabla 1. Categorías que conforman el guion de la entrevista

\begin{tabular}{|c|c|}
\hline Categoría & Contenido \\
\hline Núcleo personal & $\begin{array}{l}\text { Se consideran los elementos que se relacionan con } \\
\text { el informante clave como edad, nivel de instrucción, } \\
\text { ocupación e ideología, por mencionar algunos. }\end{array}$ \\
\hline $\begin{array}{l}\text { Organización, convocatoria digital y movili- } \\
\text { zación social }\end{array}$ & $\begin{array}{l}\text { Se busca conocer las modalidades de organización } \\
\text { de los jóvenes a través de redes sociales digitales, y, } \\
\text { a su vez, cómo estas permiten realizar actividades y } \\
\text { exponer demandas en línea. }\end{array}$ \\
\hline Valores, demandas y toma de decisiones & $\begin{array}{l}\text { Se persigue conocer la percepción de los jóvenes re- } \\
\text { lacionada con la actividad estructurante de las re- } \\
\text { des sociales digitales, la generación de interacción e } \\
\text { incidencia en las decisiones de las autoridades. }\end{array}$ \\
\hline
\end{tabular}

Fuente: Elaboración propia. 


\section{Formulación del guion}

El guion cuenta con la elaboración de categorías previamente identificadas por el investigador y que permiten la construcción del fenómeno, esto a partir de supuestos teóricos que indican la relación entre el uso de las redes sociales digitales y su efecto en la participación organizada de los jóvenes (Jara \& Saldierna, 2020). En concreto, la validación del contenido se realizó a través de la valoración de expertos que determinaron la relación entre el carácter del contenido, las categorías y el objeto de estudio (Escobar-Pérez \& Cuervo-Martínez, 2008; Pedrosa et al., 2013). Como resultado, se establecen las categorías descritas en la Tabla 1.

\section{Muestra}

La selección de los informantes calificados estuvo restringida por su disponibilidad a la participación en el estudio, esto debido a la dinámica sociopolítica en el contexto nicaragüense. Sin embargo, la intención científica del estudio, ajena al involucramiento en la política doméstica, permitió entrevistar a informantes plenamente identificados como jóvenes organizados en importantes movimientos universitarios en Nicaragua, en particular, en la Alianza Universitaria Nicaragüense y en el Movimiento UNA 19 de abril.

En consonancia con lo anterior, la muestra para la exploración del fenómeno de estudio se determinó por saturación. Sobre tal modalidad de indagación, Martínez-Salgado (2012) explica que se realizan las entrevistas hasta que la voz activa de los informantes no exhibe elementos nuevos y valiosos. En otras palabras, las entrevistas se detienen en el momento que no se perciben datos novedosos (Martínez-Salgado, 2012). Así, el muestreo estuvo acotado a la novedad de la información y saturación de esta, conformando una muestra final de 7 entrevistas a jóvenes nicaragüenses organizados en movimientos universitarios.

\section{Proceso de aplicación}

La recolección de la información tuvo lugar del 27 de abril al 25 de mayo de 2020, presentándose durante el proceso de estudio, la dificultad de contar con la intervención de los informantes calificados por la situación de confinamiento causada por la pandemia de COVID-19. Por tal motivo, las entrevistas se realizaron en línea (online) a través del servicio de Skype. Así, cada entrevista fue grabada en video y voz, previa autorización de los participantes para su posterior transcripción. La fase de transcripción fue realizada en semanas posteriores a la aplicación. 


\section{Resultados}

En primer lugar, se analizan los perfiles de los informantes calificados a través de elementos como la edad, domicilio, nivel de instrucción, ocupación, religión, ideología, entre otros (Ver Tabla 2). Esto, con el fin de contextualizar a los jóvenes que se organizan y participan en la acción colectiva digital en Nicaragua. Luego, se enfoca la atención en el análisis de la transcripción del diálogo entre investigador y entrevistado.

Tabla 2. Contexto de los jóvenes entrevistados

\begin{tabular}{|c|c|}
\hline Informante & Perfil del entrevistado \\
\hline Participante 1 & $\begin{array}{l}\text { Hombre de } 26 \text { años de edad, cristiano-católico, vive en Managua. Ha hecho } \\
\text { una pausa en la Licenciatura en Ciencias Políticas en la Universidad Poli- } \\
\text { técnica de Nicaragua. }\end{array}$ \\
\hline Participante 2 & $\begin{array}{l}\text { Mujer de } 18 \text { años de edad, vive en Managua. Es estudiante en Ciencias Po- } \\
\text { líticas. Prefiere omitir el nombre de la universidad. Ha participado como } \\
\text { voluntaria en la construcción de casas en asentamientos precarios. }\end{array}$ \\
\hline Participante 3 & $\begin{array}{l}\text { Hombre de } 29 \text { años de edad. Fue detenido producto de las manifestaciones } \\
\text { de estudiantes en 2018, por lo que omite brindar más información personal. }\end{array}$ \\
\hline Participante 4 & $\begin{array}{l}\text { Mujer de } 22 \text { años de edad, vive en Masaya. Se identifica como estudiante de } \\
\text { Comunicación social de la Universidad Centroamericana. Ha participado en } \\
\text { movimientos feministas. }\end{array}$ \\
\hline Participante 5 & $\begin{array}{l}\text { Hombre de } 24 \text { años, vive en Granada. Su carrera universitaria es Comuni- } \\
\text { cación Social, pero prefiere omitir el nombre de la universidad. Considera } \\
\text { tener una ideología centro. }\end{array}$ \\
\hline Participante 6 & $\begin{array}{l}\text { Mujer de } 22 \text { años, vive en Managua. Se identifica como estudiante de Tu- } \\
\text { rismo rural en la Universidad Nacional Agraria. Se considera de ideología } \\
\text { centro izquierda. }\end{array}$ \\
\hline Participante 7 & $\begin{array}{l}\text { Hombre de } 25 \text { años de edad, vive en Managua. Estudia y trabaja. Manifiesta } \\
\text { que es de ideología liberal. }\end{array}$ \\
\hline
\end{tabular}

Fuente: Elaboración propia. 
Como se logra observar en los perfiles de la muestra, se presenta, una mayor representación masculina. Esto no fue intencional, simplemente es resultado del proceso de acceso a los participantes a través del uso de la técnica de bola de nieve, en donde "un sujeto le da al investigador el nombre de otro, que a su vez proporciona el nombre de un tercero y así sucesivamente" (Atkinson \& Flint, 2011, p. 1). Por su parte, analizando el tipo de institución educativa, la mayoría concurre a una universidad pública, y, además, tienen su domicilio en Managua, capital de Nicaragua. Las edades de los participantes oscilan entre los 18 y 29 años de edad. Así mismo, no existe una ideología política predominante entre los jóvenes entrevistados.

Posterior a la fase de transcripción de las entrevistas, para la valoración del contenido se incorporó el texto al software de análisis NVivo 12. De manera puntual, se presenta la relación porcentual del contenido, en referencia con las categorías de análisis preestablecidas en la formulación del guion (Ver Tabla 3) y se analiza los resultados con la intervención de los niveles de abstracción, es decir, las subcategorías emergentes y organizadas a partir del procesamiento de las transcripciones.

Tabla 3. Relación entre el valor porcentual del contenido y las categorías

\begin{tabular}{lcc}
\hline Informante & $\begin{array}{c}\text { Organización, convocatoria digi- } \\
\text { tal y movilización social }\end{array}$ & $\begin{array}{c}\text { Valores, demandas y toma de } \\
\text { decisiones }\end{array}$ \\
\hline Participante 1 & $11.43 \%$ & $8.57 \%$ \\
Participante 2 & $10.00 \%$ & $15.00 \%$ \\
Participante 3 & $12.50 \%$ & $8.33 \%$ \\
Participante 4 & $9.09 \%$ & $9.09 \%$ \\
Participante 5 & $11.11 \%$ & $11.11 \%$ \\
Participante 6 & & $13.64 \%$ \\
Participante 7 & $13.64 \%$ & $12.50 \%$ \\
\hline Fuente: Elaboración propia. & & \\
\hline
\end{tabular}

Fuente: Elaboración propia. 
La Tabla 3 muestra una variación entre el 9.09\% y el 13.64\%, en lo referente a la identificación de las modalidades de organización de los jóvenes a través de redes sociales y, a su vez, cómo estas permiten realizar actividades y exponer demandas. Por su parte, la relación porcentual de la categoría de Valores, demandas y toma de decisiones se ubica entre el $8.33 \%$ y el $15 \%$. Dicho lo anterior, a continuación, se presentan los resultados más relevantes e íntimamente relacionados con las categorías deductivas.

\section{Organización, convocatoria digital y movilización social}

Iniciando con el análisis de la información, la categoría de Organización, convocatoria digital y movilización social se compone de 3 subcategorías. En primer lugar, en la subcategoría de Alcance de la convocatoria, los jóvenes miembros de movimientos universitarios entrevistados declaran un elevado alcance en la población joven, esto se debe a la trascendencia e inmediatez de las redes y porque en su mayoría son los jóvenes los que ocupan estas tecnologías:

"El alcance es a nivel nacional. En mi perfil de Facebook tengo a personas de los 15 departamentos de Nicaragua, incluyendo sus dos regiones autónomas. Con una publicación tengo alcance a todas esas personas sin necesidad de llamarlas directamente y, a su vez, estas personas comparten el contenido. Prácticamente se viraliza la convocatoria en menos de 30 minutos”

Participante 3

"La convocatoria virtual tiene mucho más alcance que una convocatoria a través de medios tradicionales porque los jóvenes pasan mucho de su tiempo en redes sociales”

Participante 6

Resulta importante señalar que la materialización de la convocatoria, es decir, la actividad presencial en un espacio público, se relaciona con las garantías de seguridad, y, en el contexto sociopolítico nicaragüense, tal presencia resulta limitada. Con todo, se expresa:

"Aunque la convocatoria tuvo un alcance de 30 mil perfiles, pero al final solo asistieron 200 personas., esto no es negativo. Es un primer paso importante, es decir, 30 mil perfiles se enteraron de la convocatoria y 200 personas se motivaron a formar parte de la movilización”

Participante 5 
Con respecto a la segunda subcategoría, que toma en cuenta a los Medios de Acción que se promueven a través del perfil del movimiento universitario en redes sociales, las acciones han evolucionado desde el 2018, cuando la iniciativa digital se concentraba únicamente en la realización de manifestaciones en el espacio público. Es así, como se practican otros medios de acción:

“... paros vehiculares, paros nacionales e incluso algunos paros de consumo”

Participante 1

“... hemos tenido reuniones con los representantes de la empresa privada en Nicaragua”

Participante 2

“... piquetes de protesta (grupo reducido de personas) en distintos puntos”

Participante 3

"Actualmente se realizan talleres virtuales y conversatorios con algunas personalidades. Se busca la alternativa digital para manifestarnos"

Participante 7

Finalmente, la subcategoría de Parámetros de la convocatoria subraya la importancia de resguardar la integridad de los manifestantes y la consideración de elementos subjetivos como el sentimiento de respaldo y confianza de los ciudadanos que se percibe en las redes.

\section{Valores, demandas y toma de decisiones}

En lo que respecta a la categoría Valores, demandas y toma de decisiones se hace notar la percepción de los jóvenes respecto a la actividad estructurante de las redes sociales, la presentación de peticiones, generación de interacción e incidencia en las decisiones de las autoridades. La primera subcategoría corresponde a Demandas en redes sociales. En particular, las demandas que más se han expuesto o se han logrado poner en relevancia desde el perfil digital de los movimientos universitarios son la libertad, la justicia y la mejora de la lógica de la democracia a través de reformas: 
"Las demandas que han tenido mayor fuerza son las demandas, por ejemplo, la libertad, el respeto a los derechos humanos, también las demandas sobre reformas que constituyen el fondo de la institucionalidad democrática del país como reformas electorales, reformas al sistema de justicia...”

Participante 1

"Definitivamente la justicia, la libertad y la democracia. En ese orden se ha expuesto, la justicia que permite a los ciudadanos alcanzar una paz relativa, el restablecimiento de las libertades públicas, económicas y sociales, junto al compromiso de reforzar la democracia”

Participante 3

"El tema de la justicia y democracia son vitales"

Participante 5

En efecto, las redes sociales permiten hacer visible distintas demandas sociales y apresurar la respuesta de los tomadores de decisiones (decision makers). Sin embargo, el especial énfasis en la mejora de la calidad democrática, refleja un alto grado de insatisfacción política, sobre todo, porque los jóvenes consideran que no se les toma en cuenta y que no se responde de manera oportuna a las necesidades de su colectivo. En ese sentido, la subcategoría de Incidencia en las autoridades refuerza la aseveración anterior. Si bien a través de las redes sociales se logra hacer visible las demandas sociales, la incidencia en las autoridades, es decir, la generación de una respuesta, se encuentra en un nivel bajo:

"El nivel de incidencia hacia las autoridades es muy poco. Te escuchan o leen tus estados y comentarios, pero solo brindan ciertas reacciones y respuestas”

Participante 2

"No se incide. A las autoridades solo les interesa tener relaciones con personas de altos rangos. No se ha creado todavía ese puente directo con los jóvenes”

Participante 4

"En las redes nos organizamos y generamos conversaciones, sin embargo, no existen canales de comunicación ni se incide directamente en las autoridades”

Participante 5 
Por su parte, la subcategoría Influencia en el interés en la política demuestra que, aunque no existe una respuesta considerable de las autoridades en las redes de Internet, el uso de las herramientas tecnológicas aumenta el interés en lo político, siempre y cuando, este tema se encuentre en el área de interés del individuo que interactúa en la plataforma, lo que reafirma la influencia de las Tecnologías de Información y Comunicación (TICs):

"Creo que la mayor parte de las personas que interactúan con las plataformas digitales de los movimientos universitarios tienen un alto interés en política nacional” Participante 1

"Si influye porque a raíz de lo que uno comunica se generan distintas reacciones y las personas tienen una idea más clara de a qué actor van a respaldar. Si no se genera esa interacción se daría pauta al desinterés y al mito”

Participante 5

\section{Discusión y conclusiones}

La presente investigación científica es un esfuerzo por determinar el papel que ejercen las redes sociales en el proceso de organización de la juventud e identificar las relaciones que se crean entre el uso de las redes sociales, el interés en lo político y la participación del ciudadano joven en Nicaragua.

Con respectó a la tecnología digital de las redes sociales y la juventud, organizada específicamente en movimientos universitarios, el uso de las redes sociales permite a los jóvenes nicaragüenses, conectarse y acercarse a un mayor número de ciudadanos, de manera fácil y rápida, esto en función de reclutar a más miembros, organizar protestas, exponer sus demandas, exigir soluciones a los problemas que atañen la vida en sociedad e impactar en la opinión pública.

Tal aseveración contesta la primera pregunta de investigación que versaba ¿Cuál es el rol que desempeña el uso de redes como generador de participación ciudadana en los jóvenes nicaragüenses?, por su parte, si bien las redes sociales inciden en el comportamiento y en el debate ciudadano, y permiten exponer las demandas como un reflejo del interés ciudadano de incidir en la toma de decisiones, las autoridades hacen caso omiso al uso multidireccional de las redes, por lo que resulta complejo dar paso a la interactividad. En otras palabras, en Nicaragua las redes sociales permiten conectar a una gran cantidad de personas que demuestran (o no) interés en los asuntos políticos, económicos y sociales, mas no conectar específicamente con las autoridades o convencer al conductor formal del Estado. Por su parte, las autoridades utilizan sus cuentas en Facebook para compartir la cotidianeidad de sus vidas y son pocas las instituciones que comunican a través de un perfil en lo digital. 
En ese sentido, el uso de las redes sociales aumenta el interés en la política, siempre que el tema político se encuentre en la zona de interés del individuo que interactúa en la plataforma virtual. Bajo esa premisa, "el uso de las redes sociales" se presenta como un estímulo que genera una respuesta, es decir, "interés en temas políticos”. Sin embargo, la tendencia de afección se encuentra moderada por la selección de contenido, afín a las preferencias individuales o sujeta al mandato de los algoritmos. Con todo, el joven motivado por el mensaje en el espacio digital utiliza las redes sociales como un instrumento que facilita la interacción y promueve la participación ciudadana no convencional. Tal condición, contesta la segunda pregunta de investigación que versaba ¿Cuál es el papel de las redes sociales en el interés político en los jóvenes nicaragüenses?

Como resultado, se esclarece el fenómeno, y en correspondencia con la evidencia teórica fundamentada, el contexto natural y la interacción con los individuos durante el proceso de investigación, se construye la conjetura: La práctica de la acción colectiva a través de nuevas tecnologías junto a la interacción social en plataformas digitales permite difundir información de fácil acceso, fomenta la organización y participación, lo que contribuye a la (re)construcción de la participación ciudadana de los jóvenes durante momentos de tensión social en Nicaragua. Ahora bien, para dar respuesta a tal conjetura resulta necesario aumentar la cantidad (sin menoscabar la calidad) de estudios científicos que hagan notar la relevancia de la tecnología y de las plataformas sociales digitales en la conversación y sofisticación política del ciudadano joven en Nicaragua.

\section{Agradecimientos}

Agradezco a la Dra. Alma Rosa Saldierna Salas por permitirme recurrir a sus conocimientos relacionados con el tema de estudio.

\section{Declaración de conflicto de intereses}

El autor no informó ningún posible conflicto de intereses.

\section{Fondos}

Este artículo científico deriva de la tesis titulada "Movimientos Universitarios, Redes Sociales y Participación Ciudadana: Incidencia de la acción colectiva virtual en los espacios de participación ciudadana en Nicaragua”, investigación apoyada por el Consejo Nacional de Ciencia y Tecnología (Conacyt) a través de una Beca Conacyt para estudios de maestría. 


\section{Referencias}

Atkinson, R., \& Flint, J. (2001). Accessing hidden and hard-to-reach populations: Snowball research strategies. Social Research Update, (33), 1-5.

Bacallao-Pino, L. (2010). Representaciones mediáticas de las redes sociales: un estudio de casos. Revista Latina de Comunicación Social, (65), 114-125. https://doi.org/10.4185/RLCS-652010-887-114-125

Bacallao-Pino, L. (2016). Redes sociales, acción colectiva y elecciones: los usos de facebook por el movimiento estudiantil chileno durante la campaña electoral de 2013. Palabra Clave 19(3), 810-937. https://doi.org/10.5294/pacla.2016.19.3.6

Banco Mundial. (2020, 3 de agosto). Nicaragua: Data source: United Nations World Population Prospects. https://datos.bancomundial.org/pais/nicaragua

Cabalin, C. (2014). Estudiantes conectados y movilizados: El uso de Facebook en las protestas estudiantiles en Chile. Revista Cientifica de Educomunicación, 22(43), 25-33. https://doi.org/10.3916/C43-2014-02

Castells, M., Fernández-Ardévol, Linchuan, J., \& Sey, A. (2007) Comunicación móvil y sociedad, una perspectiva global. Ariel - Fundación Telefónica.

Castro, I. (2018). Nicaragua: comunicación y redes en la crisis. En A. Antunes, E. De Gori, \& C. Villacorta (Eds.), Nicaragua en crisis: Entre la revolución y la sublevación (pp. 159-176). CLACSO.

Dorantes y Aguilar, G. L. (2016). Internet, sociedad y poder. Democracia digital: comunicación política en la era de la hipermediación. Universidad Nacional Autónoma de México.

Echeverria, M. (2011). ¿Apatía o desencuentro? Patrones de consumo y recepción de información política y gubernamental en jóvenes. Global Media Journal México, 8(15), 42-65.

Escobar-Pérez, J., \& Cuervo-Martínez, Á. (2008). Validez de contenido y juicio de expertos: una aproximación a su utilización. Avances en Medición, 6(1), 27-36.

Folgueiras Bertomeu, $\quad$ P. $\quad$ (2016). $\quad$ La $\quad$ entrevista. http://diposit.ub.edu/dspace/handle/2445/99003?mode=full

García, M., \& del Hoyo, M. (2013). Redes sociales, un medio para la movilización juvenil. ZER Revista de Estudios de Comunicación, 18(34), 111-125. https://doi.org/10.1387/zer.10649 
Gil de Zuñiga, H., Garcia-Perdomo, V., \& Mcgregor, S. (2015). What is second screening? Exploring motivations of second screen use and its effect on online political participation. Journal of Communication, 65(5), 793-815. https://doi.org/10.1111/jcom.12174

Jara, L., \& Saldierna, A. R. (2020). Acción colectiva virtual y espacios de participación ciudadana de los jóvenes en Nicaragua. Revista Ciencia Jurídica y Política, 6(11), 62-79. https://doi.org/10.5377/rcijupo.v6i11.11203

Martí I Puig, S. (2016). Nicaragua: Desdemocratización y caudillismo. Revista de Ciencia Política, 36(1), 239-258. https://dx.doi.org/10.4067/So718-090X2016000100011

Martínez-Salgado, C. (2012). El muestreo en investigación cualitativa: principios básicos y algunas controversias. Ciência \& Saúde Coletiva, 17(3), 613-619. https://doi.org/10.1590/S141381232012000300006

Merino, M. (2016). La participación ciudadana en la democracia. Cuadernos de divulgación de la cultura democrática. Instituto Nacional Electoral.

Muñoz, D., \& Narváez, M. (2018). ¿Cómo se informan y participan los jóvenes? Guía para organizaciones que trabajan con participación juvenil. FUNIDES.

Musso, P. (2004). A filosofía da rede. En A. Parente (Comp.), Tramas da rede: novas dimensoes filosóficas, estéticas e políticas da comunicasao (pp. 17-38). Sulina.

Osorio Mercado, H., \& Serra Vásquez, L. (2012). Cultura política de la juventud urbana de la región metropolitana de Managua. Encuentro, (91), 32-57. https://doi.org/10.5377/encuentro.voi91.679

Pedrosa, I., Suárez-Álvarez, J., \& García-Cueto, E. (2013). Evidencias sobre la Validez de Contenido: Avances Teóricos y Métodos para su Estimación. Acción Psicológica, 10(2), 3-18. http://dx.doi.org/10.5944/ap.10.2.11820

Pérez Rodríguez, R. O., González Valdivieso, M., \& Silva-Castro, M. M. (2021). Entrevistas en profundidad: técnica complementaria a las entrevistas clínicas en las intervenciones basadas en necesidades del paciente. Pharmaceutical Care España, 23(5), 34-41.

Rihawi Perez, N. (2017). El papel de las redes sociales en la cibercultura: el caso de la Primavera Árabe [Tesis de doctorado, Universidad Complutense de Madrid]. E-prints Complutense. https://eprints.ucm.es/id/eprint/47935/1/T39993.pdf

Rueda-Estrada, V. (2018). "Que se rinda tu madre": los nuevos/viejos símbolos y tácticas de la movilización social en Nicaragua. En A. Antunes, E. De Gori, \& C. Villacorta (Eds.), Nicaragua en crisis: Entre la revolución y la sublevación (pp. 91-118). CLACSO. https://doi.org/10.2307/j.ctvnpojft.8 
Sartori, G. (2009). La Democracia en 30 Lecciones. Santillana Ediciones Generales, S. L.

Serrano, A. (2015). La participación ciudadana en México. Estudios Políticos, (34), 93-116. http://dx.doi.org/10.1016/j.espol.2015.05.001

Sola-Morales, S. (2016). Las redes sociales y los nuevos movimientos estudiantiles latinoamericanos. La "Primavera Chilena” y el “\#YoSoy132". Revista Científica de Información y Comunicación, (13), 153-193. http://dx.doi.org/10.12795/IC.2016.io1.05

Telléz, D. (2009). La exclusión política de jóvenes, mujeres y pueblos indígenas: Propuestas para la Reforma Política en Nicaragua. Friedrich-Ebert-Stiftung.

Troncoso-Pantoja, C., \& Amaya-Plascencia, A. (2017). Entrevista: guía práctica para la elaboración de datos cualitativos en investigación en Salud. Revista de la Facultad de Medicina, 65(2), 329332. https://doi.org/10.15446/revfacmed.v65n2.60235

Vaccari, C., Valeriani, A., Barberá, P., Bonneau, R., Jost, J. T., Nagler, J., \& Tucker, J. A. (2015). Political expression and action on social media: Exploring the relationship between lowerand higher-threshold political activities among Twitter users in Italy. Journal of ComputerMediated Communication, 2O(2), 221-239. https://doi.org/10.1111/jcc4.12108

Valencia, J., \& García, C. (2014). Movimientos sociales e internet. Editorial Pontificia Universidad Javeriana.

Valenzuela, S., Arriagada, A., \& Scherman, A. (2012). The social media basis of youth protest behavior: The case of Chile. Journal of Communication, 62(2), 299-314. https://doi.org/10.1111/j.1460-2466.2012.01635.x

Vargas-Jiménez, I. (2012). La entrevista en la investigación cualitativa: nuevas tendencias y retos. Calidad en la Educación Superior, 3(1), 119-139. https://doi.org/10.22458/caes.v3i1.436 